\title{
Agir au lieu de subir
}

\section{Yvonne Gillia, Lucas Schult ${ }^{b}$}

a Dre méd., membre du Comité central de la FMH, responsable du département Numérisation / eHealth

${ }^{b}$ Lucas Schult: Directeur général (CEO) de Health Info Net AG (HIN), Wallisellen

L'introduction du dossier électronique du patient (DEP) se fait attendre. Cela n'est pas forcément un mal, car son report est l'occasion d'apporter de nouvelles améliorations du point de vue des spécialistes. Par ailleurs, dans le cadre du DEP et en complément de celui-ci, il existe également d'autres offres de médecins axées sur le bénéfice médical.

Nous sommes entrés dans l'ère des dossiers électroniques des patients (DEP). Aux termes de la loi fédérale sur le dossier électronique du patient (LDEP), les hôpitaux de soins aigus, les cliniques de rééducation et les établissements de psychiatrie stationnaire doivent proposer le DEP depuis le 15 avril 2020. En théorie. Car dans les faits, le DEP n'est pas encore opérationnel - et ne devrait pas l'être d'ici à 2021 -, raison pour laquelle sa manifestation reste très discrète pour l'instant, si ce n'est au travers d'une incertitude généralisée.

Selon les dires, le retard du lancement du DEP serait dû à la complexité de la certification des communautés de référence et à l'accréditation probablement non moins complexe des certificateurs. En tout état de cause, le report du lancement offre la possibilité de corriger, pour ainsi dire dans la dernière ligne droite, un certain nombre de défauts de conception du DEP [1].

\section{Le dossier du patient}

Commençons par lever un malentendu. Comme son nom l'indique, le DEP a été conçu par le législateur

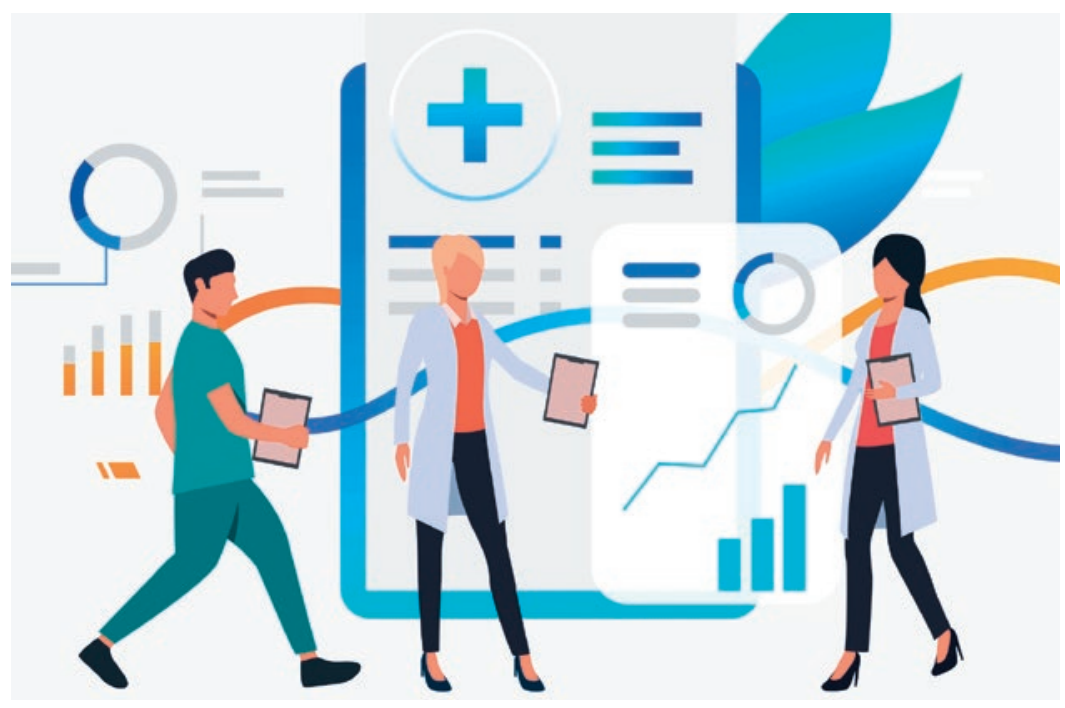

comme un dossier de patient [2]. Il vise à accorder aux patients la souveraineté sur leurs propres données, lesquelles étaient jusqu'à présent stockées dans les armoires à classeurs et les ordinateurs des prestataires de services - certes sous bonne garde, mais sans aucune transparence et difficilement accessibles pour les patients. Avec le DEP, les patients pourront contrôler eux-mêmes l'accès à leurs documents médicaux et «consulter les informations accessibles dans le DEP, se poser des questions et les régler avec les professionnels de la santé» [3]. En effet, l'encouragement à la compétence des patients en matière de santé se trouve être aussi l'un des cinq objectifs de la politique de santé de la Stratégie eHealth Suisse 2.0 [4].

Jusqu'ici, tout va bien. Mais cela montre également que le DEP, en tant que système secondaire centré sur le patient, n'est a priori pas un instrument destiné au corps médical. Le DEP ne prévoit ni services supplémentaires spécialement adaptés aux professionnels de la santé, ni données structurées, ni intégration en profondeur avec le système primaire du médecin [5]. C'est ce qui explique l'absence même d'un financement réglementé - selon eHealth Suisse, les fournisseurs de DEP doivent «trouver eux-mêmes un financement viable pour le DEP» [6].

\section{Bénéfice pour le corps médical et les patients}

Cette contribution ne saurait en aucun cas être comprise comme un plaidoyer contre un DEP centré sur le patient. Nous tenons au contraire à souligner que le DEP n'apportera aux patients le bénéfice souhaité que s'il génère par ailleurs de la valeur ajoutée pour les professionnels de la santé et qu'il influe positivement sur la qualité et l'efficacité des soins médicaux, par exemple en facilitant la collaboration interdisciplinaire et interprofessionnelle. 
Dans le passé, la FMH a rappelé à plusieurs reprises que le DEP aurait un bénéfice très limité - du moins dans sa forme actuelle - pour les fournisseurs de prestations. Rétrospectivement, force est de constater que le corps médical a longtemps observé l'élaboration du DEP de l'extérieur et a eu besoin de temps pour trouver les instruments idoines lui permettant d'apporter sa pierre à

\section{Le DEP n'apportera aux patients le bénéfice} souhaité que s'il génère par ailleurs de la valeur ajoutée pour les professionnels de la santé.

l'édifice. Un autre facteur aggravant est l'approche «top down» adoptée par les autorités dans la mise en œuvre, qui exclut de fait les médecins praticiens tant dans le domaine de la gestion stratégique que dans celui de la conduite opérationnelle.

\section{Une cybersanté sans fournisseurs de prestations ambulatoires?}

La première Stratégie eHealth Suisse de la Confédération, adoptée en 2007, a défini la vision selon laquelle le patient doit pouvoir, au moyen de ressources informatiques adaptées, «accéder à tout moment et en tout lieu à d'importantes informations pertinentes sur sa personne et bénéficier de prestations de leur part» et "participer activement aux décisions concernant son comportement et ses problèmes de santé». Il est aisé de voir là l'essence même du DEP actuel. La stratégie fait l'impasse sur la question de savoir comment le corps médical indépendant devrait être intégré dans le nouveau paysage émergent des systèmes et des processus.

Or, dès le début du millénaire, il était de plus en plus manifeste que, dans un grand nombre de projets de cybersanté initiés par des cantons ou des hôpitaux, le point de vue des fournisseurs de prestations ambulatoires n'était toujours pas pris en compte. Les médecins indépendants risquaient d'être pris en tenaille entre la politique et l'informatique, voire d'être les laissés-pour-compte des avancées dans ce domaine. C'est pourquoi un projet pilote exploratoire - baptisé «Ponte Vecchio» - a été lancé en 2012 dans le canton de Saint-Gall avec les assurés Managed Care et le cas d'application «Rapport de sortie», lequel met explicitement l'accent sur les fournisseurs de prestations ambulatoires. Dans ce cadre, un groupe de travail a en outre été créé en 2014 afin d'identifier précisément les données "pertinentes pour le traitement» (p. ex. les rapports sur l'état des plaies dans le rapport de sortie) une question qui nous préoccupe encore aujourd'hui dans le cadre du DEP [7].
Dans le même temps, en Suisse romande, le projet pilote "e-toile», déjà plus ambitieux, a été intégré dans "MonDossierMedical». Aujourd'hui, l'Université de Genève propose d'ores et déjà une formation postgraduée pour les médecins titulaires d'un master en informatique médicale.

\section{De «Ponte Vecchio» à AD Swiss}

Le laboratoire expérimental «Ponte Vecchio» avait également pour mission de montrer si le HIN propre aux médecins était en mesure d'exploiter une infrastructure de base de cybersanté pour le corps médical indépendant. La nécessité d'une telle infrastructure ne fait pas débat - et ne l'a jamais fait - étant donné que les investissements des cabinets individuels ou de groupe requis ne peuvent être financés par les seuls cabinets individuels ou de groupe [8]. La conclusion du projet «Ponte Vecchio" selon laquelle HIN n'était pas non plus en mesure de mettre sur pied une telle infrastructure en l'absence de partenaires a finalement conduit à la fondation d'AD Swiss avec pour objectif l'autodétermination des fournisseurs de prestations ambulatoires en matière de participation à l'avenir numérique.

Toujours en 2014, le Groupe de travail interprofessionnel sur le dossier électronique du patient (GTIP DEP) a été constitué à l'initiative de la FMH, au moment où le constat s'est finalement imposé qu'il n'était pas souhaitable de s'en remettre à la seule mise en œuvre technique et qu'il était nécessaire d'asseoir le DEP sur une large adhésion des spécialistes. Le GTIP (IPAG) s'est

En 2014, le Groupe de travail interprofessionnel sur le dossier électronique du patient a été constitué à l'initiative de la FMH.

donné pour mission de coordonner et de mutualiser les besoins des différents groupes professionnels issus de huit associations dans le domaine de la cybersanté et de contribuer au lancement d'un DEP bénéfique au moyen de solutions communes. Et le succès fut au rendez-vous, comme le montrent les rapports sur la cybermédication et la «Transition of Care» (eToC), qui ont été remis à eHealth Suisse fin 2015 [9].

\section{Le corps médical peut et doit apporter sa pierre à l'édifice}

Les exemples mentionnés (à côté de beaucoup d'autres) montrent que le corps médical - seul ou avec des partenaires - est apte à exercer une influence significative. Cela vaut également pour les fournisseurs de prestations ambulatoires. C'est pourquoi la participation des 


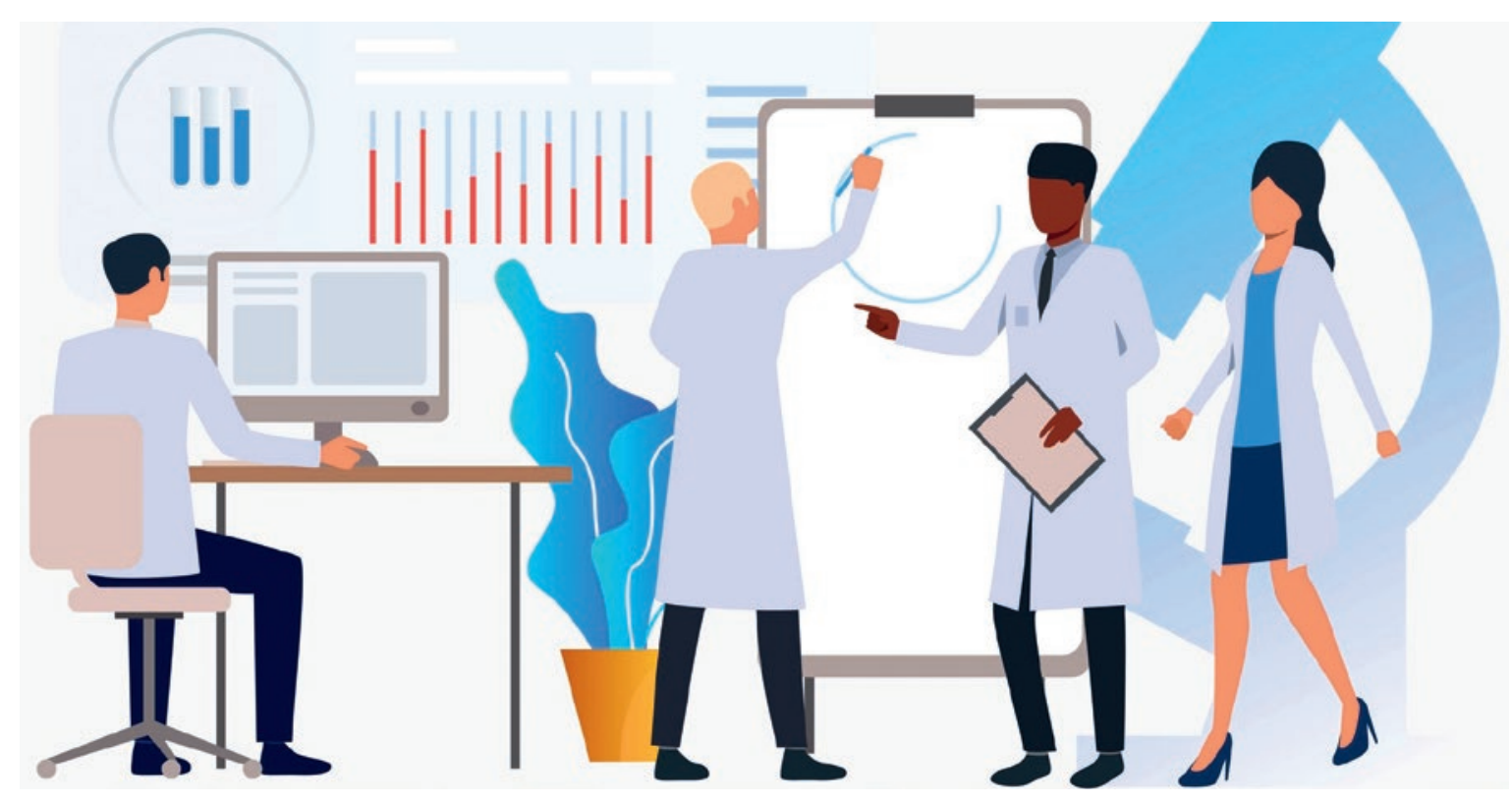

médecins indépendants au DEP est possible d'emblée quand bien même sur une base volontaire dans un pre-

Crédit photo

(c) Mstjahanara903 | Dreamstime.com mier temps. En effet, par leur participation, ils bénéficient non seulement d'une longueur d'avance en termes de connaissances, mais aussi de la possibilité de contribuer à donner corps au projet.

Là où les cas d'utilisation (p. ex. eMediplan, ordonnance électronique) sont encore trop peu tournés vers la réalité du travail des médecins praticiens, des comités de prestataires de services et de fournisseurs de systèmes primaires, tels que le cercle de travail d'AD Swiss compétent, œuvrent d'ores et déjà à la simplification des processus par le biais de connexions intégrées [10]. En outre, la Communauté DEP AD Swiss garantit un accès à bas seuil et à coût avantageux au DEP ainsi que de précieux services supplémentaires. La Communauté, comme toutes les communautés (de référence), est actuellement en cours de certification. Parallèlement, à titre d'exemple éloquent, rappelons qu'un pont vers la Suisse romande est en train d'être jeté afin de lever tout obstacle susceptible d'entraver la collaboration entre $\mathrm{AD}$ Swiss et les communautés de référence. A notre avis, les médecins en particulier peuvent - y compris dans l'intérêt de leurs patients et en tant que personnes de confiance - devenir une force motrice dans le domaine de la cybersanté en s'appropriant le DEP et/ ou les "services complémentaires» comme un instrument de l'activité médicale et en fixant le bon cap pour le développement de leur point de vue de spécialistes. Personne ne sait si le DEP sera un jour couronné de succès. Toutefois, la roue de la «cybersanté» ne peut pas reculer. Le corps médical a la possibilité, ici et maintenant, d'influencer cette évolution dans son propre intérêt.

\section{Références}

1 Yvonne Gilli: Un retard qui a du bon. Bulletin des médecins suisses 2020;101(36):1056 (DOI: https://doi.org/10.4414/saez.2020.19184)

2 Outre la qualité des traitements, la sécurité des patients et l'efficacité, l'article énonçant le but de la LDEP mentionne explicitement la promotion des "compétences des patients en matière de santé»: loi fédérale sur le dossier électronique du patient (LDEP) du 19 juin 2015 (état au 15 avril 2020) (https://www.admin.ch/opc/fr/classified-compilation/20111795/index.html)

3 Tania Weng-Bornholt, Urs Zanoni et al.: Livre blanc «Compétences en matière de santé et dossier électronique du patient: comment les communautés de référence peuvent promouvoir un DEP répondant aux besoins de la population locale». Aarau 2019 (https:// www.e-health-suisse.ch/fileadmin/user_upload/Dokumente/F/ livre-blanc-competence-en-sante-dep.pdf)

4 Stratégie eHealth Suisse 2.0 2018-2022. Objectifs et mesures de la Confédération et des cantons en vue de la diffusion du dossier électronique du patient et de la coordination de la numérisation du dossier électronique du patient. Berne 2018 (https://www.e-health-suisse.ch/fileadmin/user_upload/Dokumente/2018/F/181214_Strategie-eHealth-Suisse-2.0_f.pdf)

5 Les exigences minimales fixées par le législateur impliquent un accès basé sur un portail, c'est-à-dire l'existence d'un site Web. A contrario, le personnel de santé a besoin d'une intégration en profondeur avec les systèmes primaires existants.

6 https://www.patientendossier.ch/fr/professionnels-de-la-sante/ informations/questions-frequentes/combien-coute-le-dep (consulté le 29.9.2020)

7 Yvonne Gilli: La question des responsabilités, obstacle à la mise en œuvre du DEP? Bulletin des médecins suisses 2019;100(48):1600 (https://bullmed.ch/article/doi/saez.2019.18444)

8 Urs Stoffel: Pour un avenir numérique de la communauté médicale. Bulletin des médecins suisses 2014;95(33):1171 (DOI: https://bullmed. ch/article/doi/saez.2014.02896

9 Gert Printzen: IPAG - collaboration interprofessionnelle pendant l'année du singe. Bulletin des médecins suisses 2016;97(O2):45 (DOI: https://bullmed.ch/article/doi/saez.2016.04350)

10 L'AD Swiss Community (ADSC) rassemble des prestataires et des fournisseurs de services techniques, médicaux et administratifs. L'association s'entend comme comme un incubateur pour la mise en œuvre de projets de numérisation praticables (https://www. ad-swiss.ch/fr/verein-ad-swiss-community/). 